\title{
Evaluation of Anthracnose Resistance among Tea Genetic Resources by Wound-Inoculation Assay
}

\author{
Katsuyuki YOSHIDA* and Yoshiyuki TAKEDA \\ Department of Tea, National Institute of Vegetable and Tea Science \\ (Makurazaki, Kagoshima 898-0087, Japan)
}

\begin{abstract}
Tea anthracnose, caused by Colletotrichum theae-sinensis (Miyake) Yamamoto, is one of the most serious diseases of cultivated tea (Camellia sinensis L.) in Japan. In the present study, we evaluated tea plants genetically resistant to anthracnose by using a novel wound-inoculation assay. Conidia of $C$. theae-sinensis were suspended in an adhesive mixture consisting of potato-sucrose broth and methylcellulose $400 \mathrm{cP}(3 \% \mathrm{w} / \mathrm{v})$. Detached mature tea leaves were wounded crosswise by a screwdriver with an adhesive conidial suspension. Inoculated leaves were cultivated on ROOTCUBES ${ }^{\circledR}$ growing medium for 2 weeks in a growth chamber. The degree of resistance was estimated from lesion size. Five hundred tea genetic resources at the Makurazaki Tea Research Station were assayed in screening for anthracnose-resistant tea plants. Most of the foreign-introduced tea plants showed resistance, whereas native Japanese tea plants showed wide variation in the resistance to anthracnose. These results suggest that crossbreeding of native Japanese tea cultivars with foreign introduced varieties will be useful for breeding anthracnose-resistant tea plants of suitable quality.
\end{abstract}

Discipline: Tea breeding / Plant disease

Additional key words: breeding, Camellia sinensis, Colletotrichum theae-sinensis

\section{Introduction}

Tea (Camellia sinensis (L.) O. Kuntze) is one of the most important cash crops in the warm southwest areas of Japan. Green tea had been cultivated by planting tea seedlings before the 1950s. There were many tea plants which had different genetic backgrounds in the same tea field at that time. After that, the cutting propagation of tea was developed and the green tea cultivar 'Yabukita' has been cultivated instead of tea-seedlings since the 1970s. 'Yabukita' can be propagated easily from cuttings and the high quality of its tea has resulted in its being cultivated in over $70 \%$ of all tea fields in Japan. Monoculture cultivation systems of 'Yabukita' could be harvested for new tea shoots synchronously and could make possible the consistent quality of green tea. However, this has been the cause of severe pest problems such as outbreaks of tea anthracnose caused by Colletotrichum theaesinensis (Miyake) Yamamoto. Tea anthracnose has been known as a tea disease in Japan for a long time. 'Yabukita' is susceptible to anthracnose, therefore, the tea anthracnose problem has been serious since the 1980s.

Outbreaks of anthracnose occur from summer to autumn. The tea anthracnose fungus penetrates into new shoots from trichomes ${ }^{2}$, and the infection hyphae develop vascular bundles ${ }^{1}$. The lesion of anthracnose appears 14 to 20 days after trichome infection and infected leaves are withered by the damage of lesion development ${ }^{1}$. This disease reduces the depth of the leaf layer and the number of branches of tea bushes, resulting in a decreased first flush on green tea plants ${ }^{1}$. Therefore, repeated spraying with fungicides is needed to protect tea plants against anthracnose. However, fungicide-resistant mutants of anthracnose fungi have emerged due to over-spraying with fungicides since the $1980 \mathrm{~s}^{6}$. Moreover, consumers have become more interested in food safety and want to buy chemical-free green tea in recent years. To reduce the use of chemicals in tea cultivation, it is important to breed anthracnose-resistant tea cultivars. However, a convenient, reliable and reproducible resistance assay for tea anthracnose had not been available for a long time.

We observed natural occurrences of anthracnose in 2,392 tea genetic resources at the Makurazaki Tea Research Station of the National Institute of Vegetable and Tea Science by counting infected leaves of each plant $^{8}$. While this method is convenient, the resistance is affected by weather and cultivation conditions. Yamagu-

*Corresponding author: fax +81-993-76-2264; e-mail gohteny@affrc.go.jp

Received 30 September 2005; accepted 6 January 2006. 
chi and Ando developed a wound-inoculation resistance assay for tea anthracnose ${ }^{11}$. This method was used on new tea shoots for the resistance assay, but the results of their wound-inoculation assay didn't match the results of field observations. Therefore, we recently developed a novel method to assay the anthracnose resistance in tea plants $^{13}$. In the present study, we assayed the anthracnose resistance of 500 tea genetic resources to identify the genetic variation of anthracnose resistance and to screen for breeding materials of pest-resistant tea.

\section{Materials and methods}

\section{Plant Material}

The Makurazaki Tea Research Station of the
National Institute of Vegetable and Tea Science has collected tea genetic resources from tea growing areas domestically and abroad. About 5,000 tea genetic resources have been collected and planted at the Makurazaki Tea Research Station. Five hundred tea genetic resources were used in this study. All tea genetic resources were grown in a tea field for at least 5 years. Makurazaki is the northern limit for growing most of the Assam varieties in outdoor fields in Japan.

Tea genetic resources were classified into three groups. Group I consists of 200 tea genetic resources of Camellia sinensis var. assamica (Assam variety). These tea seeds were introduced from India, Sri Lanka, Iran, Taiwan, the former Soviet Union, and countries of South East Asia (Table 1). Group II consists of 158 foreign-

Table 1. Abbreviations and origins of tested tea genetic resources in this study

\begin{tabular}{|c|c|c|c|}
\hline Abbreviation & Origin & Introduced year & Classification \\
\hline Abo & Malaysia & 1955 & A \\
\hline $\mathrm{Ai}$ & Assam, India & 1929 & A \\
\hline $\mathrm{Aj}$ & Indonesia & 1934 & A \\
\hline $\mathrm{AK}$ & Darjeeling, India & 1931 & A \\
\hline Boh & Malaysia & 1956 & A \\
\hline Bum & Myanmar & 1954,1963 & A \\
\hline $\mathrm{Ct}$ & Taiwan & 1961 & A \\
\hline IND & India & 1965 & A \\
\hline PKS & Bangladesh & 1965 & A \\
\hline Chahon-Shan, Shan & Vietnam & 1961 & A \\
\hline SRL & Candy, Sri Lanka & 1962 & A \\
\hline Stock & India & 1955 & A \\
\hline Taiwanyamacha & Taiwan & 1969,1978 & A \\
\hline Chahon-Ast & India & 1972 & A \\
\hline IRN & Iran & 1973 & $\mathrm{C}$ \\
\hline $\mathrm{MC}$ & Former Soviet Union & 1936,1969 & $\mathrm{C}$ \\
\hline Gruszynski & Former Soviet Union & 1964 & $\mathrm{~B}(\mathrm{~A}, \mathrm{C}, \mathrm{S})$ \\
\hline Makura-Cd & Darjeeling, India & 1954,1968 & $\mathrm{~S}$ \\
\hline Makura-Ck & Anhui Province, China & 1937 & $\mathrm{~S}$ \\
\hline Makura-Cm & Anhui Province, China & 1937 & $\mathrm{~S}$ \\
\hline Makura-Cn & Jiangxi Province, China & 1937 & $\mathrm{~S}$ \\
\hline Makura-Cp & Zhejiang Province, China & 1937 & $\mathrm{~S}$ \\
\hline $\mathrm{Ch}$ & Hubei Province, China & 1891 & $\mathrm{~S}$ \\
\hline Miya-Ck & Anhui Province, China & 1964 & $\mathrm{~S}$ \\
\hline K & Korea & 1972,1973 & $\mathrm{~S}$ \\
\hline Cultivar of China & China \& Taiwan & - & $\mathrm{C}$ \\
\hline Kanaya --gou & Breeding line of Kanaya & - & $\mathrm{B}(\mathrm{A}, \mathrm{C}, \mathrm{S})$ \\
\hline Makurazaki --gou & Breeding line of Makurazaki & - & $\mathrm{B}(\mathrm{A}, \mathrm{C}, \mathrm{S})$ \\
\hline Miyazaki --gou & Breeding line of Miyazaki Pref. & - & $\mathrm{B}(\mathrm{A}, \mathrm{C}, \mathrm{S})$ \\
\hline Saitama --gou & Breeding line of Saitama Pref. & - & $\mathrm{B}(\mathrm{C}, \mathrm{S})$ \\
\hline
\end{tabular}

A: C. sinensis. var. assamica; S: C. sinensis var. sinensis; B: Breeding Line; C: Crossbreed of A and S. Kanaya and Makurazaki: Tea research stations of the National Institute of Vegetable and Tea Science. Miyazaki and Saitama: The prefectural tea research stations. 
introduced tea genetic resources of Camellia sinensis var. sinensis (introduced Chinese variety). These tea seeds were introduced from China, Korea, Taiwan, and India (Table 1). The genetic resources of Gruszynski were breeding lines that were introduced from the former Soviet Union. These breeding lines contain crossbreeds of Assam varieties, crossbreeds of introduced Chinese varieties and Japanese cultivars. Foreign introduced tea genetic resources were selected by the differences in collected country and tea quality. Group III consists of 142 Japanese tea cultivars, plants of breeding lines and native tea plants that were collected from across Japan (Table 1). Some of these Japanese tea genetic resources are crossbreeds between Japanese native tea plants and Assam or introduced Chinese varieties.

\section{Pathogen}

Colletotrichum theae-sinensis (Miyake) Yamamoto, isolate SI-4-2, was used in this study. This fungus was cultivated on autoclaved tea leaves in an Erlenmeyer flask for a month at $26^{\circ} \mathrm{C}^{13}$. Conidia of the fungus were washed from tea leaves with sterile distilled water and the conidial suspension was filtered with four-layer gauze. Conidia were collected by centrifugation at $3,800 \times \mathrm{g}$ for 2 min. Pellets of conidia were re-suspended in sterile distilled water and their conidial concentration was calculated by hemacytometer. For wound-inoculation, an adhesive conidial suspension was prepared ${ }^{13}$. Conidia were suspended in a potato-sucrose broth containing $3 \%$ $(\mathrm{W} / \mathrm{V})$ methyl-cellulose $400 \mathrm{cP}$. The conidial concentration was adjusted to $1 \times 10^{7}$ spores $/ \mathrm{mL}$.

\section{Wound-inoculation assay}

A diagram of the wound-inoculation assay is shown in Fig. 1. Six mature tea leaves of each genetic resource were harvested from the tea field and washed with neutral detergent and distilled water. Washed leaves were wounded crosswise by a screwdriver with an adhesive conidial suspension. Inoculated tea leaves were incubated on moist paper towels at $26^{\circ} \mathrm{C}$ in the dark for overnight. Finally, tea leaves were planted on a ROOTCUBES ${ }^{\circledR}$ Growing Medium (Smithers-Oasis Co.), covered with a plastic sheet, and kept for 2 weeks under high humidity in a growth chamber $\left(26^{\circ} \mathrm{C}, 10,000\right.$ lux, 12 $\mathrm{h}$ light/12 h dark).

Inoculation tests of each plant were repeated twice, and the mean values of lesion size were determined. The degree of anthracnose resistance was estimated from lesion size ${ }^{13}$. Anthracnose resistance was classified into four grades, i.e. susceptible (lesion $>8 \mathrm{~mm}$ ), moderately resistant ( 5 to $8 \mathrm{~mm}$ ), resistant ( 3 to $5 \mathrm{~mm}$ ) and highly resistant $(<3 \mathrm{~mm})$. Representative lesion formations in susceptible, moderately resistant and resistant tea plants are shown in Fig. 2.

\section{Results}

\section{Variation of anthracnose resistance among the genetic resources of Camellia sinensis var. assamica \\ Ninety-one Assam varieties showed high resistance,} 95 plants showed resistance, 14 plants showed moderate resistance, and no plants were susceptible (Table 2). The pure Assam varieties such as Abo, Ai, Ak, Aj, ChahonShan, IND, PKS, SRL, and Shan were either resistant or highly resistant. The resistance of these plants was not related to the country of origin. Genetic resources of IRN, MC, and Taiwanyamacha showed wide variation of resistance against anthracnose, however, no plant showed susceptibility. These genetic resources were contained in crosses between the C. sinensis var. assamica and C. sinensis var. sinensis.

\section{Variation of anthracnose resistance among introduced Chinese varieties}

Fifty-one plants showed high resistance, 94 showed resistance, 11 showed moderate resistance, and 2 were susceptible (Table 3). Genetic resources of Makura-Cd and Miya-Ck showed wide variation of resistance. 'Makura-Cd109' and 'Miya-Ck68' showed susceptibility like 'Yabukita'. Genetic resources of Makura-Ck, Makura-Cm, Makura-Cn, and Makura-Cp showed variations of resistance, however, no plant showed susceptibility. Genetic resources of $\mathrm{Ch}$ showed resistance and moderate resistance. All plants of $\mathrm{K}$ showed resistance. Green tea cultivars introduced from China (such as Chin Shin Dah Pan) showed resistance and moderate resistance (Ying Jy Horong Shin). Some genetic resources of Gruszynski were bred from introduced Chinese varieties and they showed anthracnose resistance.

\section{Variation of anthracnose resistance among Japanese tea genetic resources}

The classification of anthracnose resistance among Japanese tea genetic resources is shown in Table 4. Japanese tea genetic resources include native Japanese plants, crosses between native Japanese plants, and crosses between native Japanese plants and the Assam or introduced Chinese varieties. Japanese tea genetic resources showed a wide range of anthracnose resistance. Fifteen of the Japanese tea genetic resources showed high anthracnose resistance. Most of these tea plants, however, were crosses between native Japanese plants and the Assam variety or introduced Chinese varieties. Two pure native Japanese tea plants of 'Yamatomidori' and 'Tsukasami- 
(1) Detached mature tea leaves are wounded crosswise by a screwdriver with an adhesive conidial suspension.

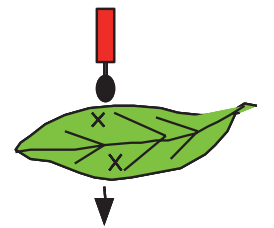

(2) Inoculated tea leaves are incubated on moist paper towels at $26^{\circ} \mathrm{C}$ in the dark for overnight.

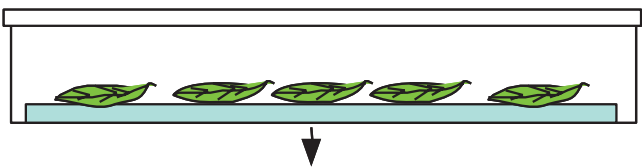

(3) Tea leaves are cultivated on $\mathrm{ROOTCUBE}^{\circledR}$ growing medium in a small moist chamber. It is set in a growth chamber $\left(26^{\circ} \mathrm{C}, 10,000\right.$ lux, $12 \mathrm{~h}$ light $/ 12 \mathrm{~h}$ dark) for 2 weeks.

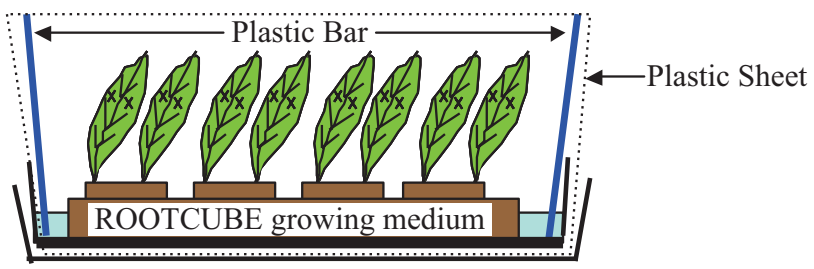

A plastic sheet is sandwiched between two plastic vats.

Fig. 1. Diagram of wound-inoculation assay for tea anthracnose resistance

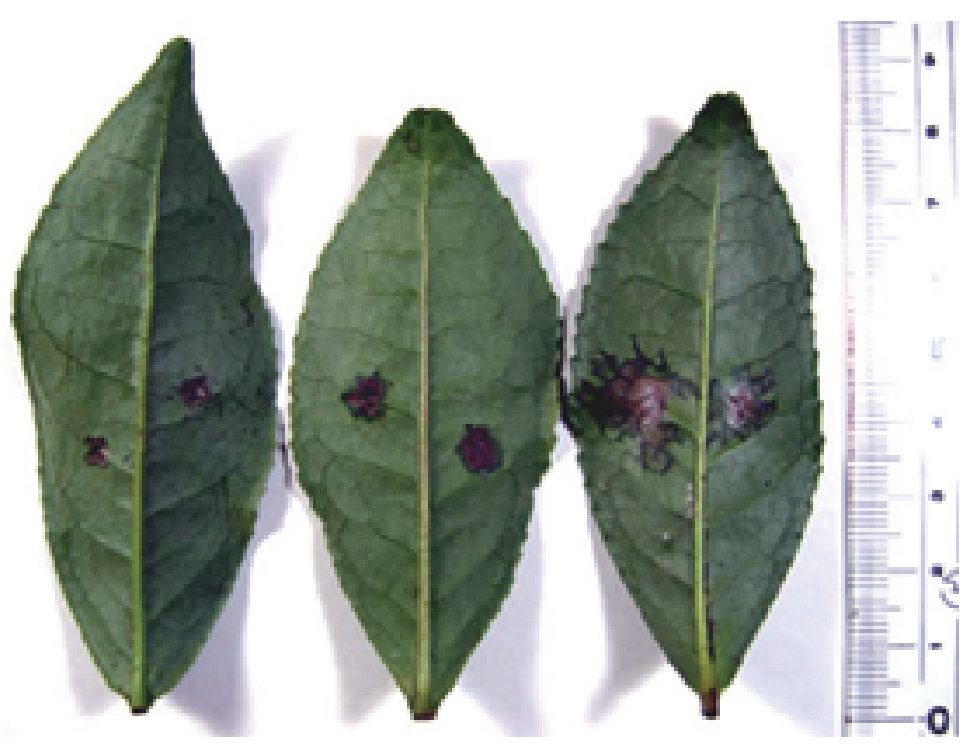

Fig. 2. Typical anthracnose lesion formation of tea with different degrees of resistance This photo was taken 2 weeks after inoculation.

Left: 'Minamisayaka' (resistant), center:'Musashikaori' (moderately resistant), right: 'Sakimidori' (susceptible). 
Table 2. Classification of anthracnose resistance among tea genetic resources of $C$. sinensis var. assamica

Highly resistant (lesion $<3 \mathrm{~mm}), \mathrm{n}=91$

Abo12, Abo18, Abo22, Abo23, Abo24, Ai1, Ai6, Ai8, Ai9, Ai16, Ai26, Ai37, Ai39, Ai66, Aj2, Ak21, AK40, Ak220, Ak226, Ak271, Ak272, Ak360, Ak568, Ak571, Ak1333, AK1440, AK1699, Ak1823, CA288, Chahon-Shan16, Chahon-Shan21, ChahonShan32, Chahon-Shan35, Chahon-Shan39, Chahon-Shan40, Gruszynski5, IND8, IND9, IND10, IND18, IND75, IND76, IND77, IND79, IND100, IND102, IRN1, IRN5, IRN7, IRN9, IRN10, IRN20, IRN21, MC3, PKS96, PKS97, PKS116, PKS224, PKS247, PKS274, PKS283, PKS292, PKS349, PKS411, PKS423, PKS434, PKS438, Shan, SRL1, SRL2, SRL3, SRL5, SRL10, SRL15, SRL16, SRL17, SRL19, SRL30, SRL33, SRL37, SRL40, SRL80, SRL85, Taiwanyamacha1, Taiwanyamacha2, Taiwanyamacha5, Taiwanyamacha6, Taiwanyamacha10, Taiwanyamacha13, Taiwanyamacha15, Taiwanyamacha18

Resistant (lesion 3 to $5 \mathrm{~mm}$ ), $\mathrm{n}=95$

Abo2, Abo3, Abo4, Abo27, Ace7, Ace14, Ace37, Ai2, Ai4, Ai7, Aj1, Aj11, Aj39, Ak9, Alu6, Chahon-Ast37, Boh330, Chahon-F4, Chahon-Shan5, Chahon-Shan37, Stock172, Stock173, BUM1, BUM2, BUM3, BUM4, BUM5, BUM6, BUM7,Ct5316, Ct5327, Ct5336, Ct5606, Ct5607, Ct5610, Ct5615, Gruszynski1, Gruszynski2, Gruszynski9, IND1, IND2, IND3, IND4, IND7, IRN2, IRN3, IRN4, IRN6, IRN8, IRN12, IRN13, IRN14, IRN15, IRN24, IRN25, IRN26, IRN28, IRN34, IRN36, IRN38, IRN41, IRN42, MC6, MC14, MC17, MC19, MC20, MC24, MC28, MC29, MC30, MC31, MC33, PKS8, PKS15, PKS106, PKS108, PKS112, PKS109, Taiwanyamacha7, Taiwanyamacha8, Taiwanyamacha9, Taiwanyamacha12, Taiwanyamacha14, Taiwanyamacha16, Taiwanyamacha19, Taiwanyamacha23, Taiwanyamacha70, Taiwanyamacha71, Taiwanyamacha75, Taiwanyamacha76, Taiwanyamacha95, Taiwanyamacha82, Taiwanyamacha83, Taiwanyamacha84

Moderately resistant ( 5 to $8 \mathrm{~mm}), \mathrm{n}=14$

Ct5604, IRN11, IRN23, IRN30, IRN33, IRN40, MC1, MC8, MC12, MC25, MC43, Taiwanyamacha72, Taiwanyamacha80, Taiwanyamacha81

No plants showed susceptibility.

Table 3. Classification of anthracnose resistance among tea genetic resources of introduced Chinese varieties

Highly resistant (lesion $<3 \mathrm{~mm}), \mathrm{n}=51$

Kana-Ck17, Makura-Cd3, Makura-Cd6, Makura-Cd7, Makura-Cd16, Makura-Cd18, Makura-Cd17, Makura-Cd86, Makura-Cd106, Makura-Cd105, Makura-Ck4, Makura-Ck6, Makura-Ck9, Makura-Ck10, Makura-Ck11, Makura-Ck12, Makura-Ck13, MakuraCk15, Makura-Ck16, Makura-Ck20, Makura-Ck21, Makura-Ck24, Makura-Cm29, Makura-Cm30, Makura-Cm31, Makura-Cm34, Makura-Cm37, Makura-Cn2, Makura-Cn5, Makura-Cn10, Makura-Cn13, Makura-Cn38, Makura-Cn39, Makura-Cn44, MakuraCn94, Makura-Cp1, Makura-Cp4, Makura-Cp8, Miya-Ck4, Miya-Ck6, Miya-Ck10, Miya-Ck20, Miya-Ck32, Miya-Ck33, MiyaCk34, Miya-Ck35, Miya-Ck36, Miya-Ck37, Miya-Ck48, Miya-Ck65, Miya-Ck70

Resistant (lesion 3 to $5 \mathrm{~mm}$ ), $\mathrm{n}=94$

Tiee Guan In, Chin Shin Oolong, Hwang Gan, Wuu Yi, San Cha Jy Lan, Chin Shin Dah Pan, Dah Yeh Oolong, Jwu Yeh, Benibana, Karabeni, Chahon-C5, Chahon-C17, Ch5343, Ch5346, Ch5349, Ch5355, Ch5360, Ch5361, Ch5363, Ch5364, Ch5402, Ch5403, Ch5412, Gruszynski4, Gruszynski6, Gruszynski7, Gruszynski10, K4, K8, K9, K15, K20, K21, K22, K23, K27, K28, K30, K31, K34, K35, K36, K38, K39, K41, K42, K44, K45, K52, K53, K54, Makura-Cd4, Makura-Cd5, Makura-Cd8, Makura-Cd9, MakuraCd21, Makura-Cd50, Makura-Cd110, Makura-Ck5, Makura-Ck17, Makura-Ck18, Makura-Ck23, Makura-Ck25, Makura-Ck30, Makura-Ck31, Makura-Ck32, Makura-Ck35,Makura-Cm10, Makura-Cm11, Makura-Cm12, Makura-Cm14, Makura-Cm21, Makura-Cm26, Makura-Cn21, Makura-Cn25, Makura-Cn27, Makura-Cn56, Makura-Cn87, Makura-Cn90, Makura-Cn66, MakuraCn91, Makura-Cp3, Makura-Cp12, Makura-Cp13, Makura-Cp20, Makura-Cp25, Makura-Cp26, Makura-Cp28, Miya-Ck2, MiyaCk19, Miya-Ck26, Miya-Ck79, Miya-Ck80, Miya-Ck91

Moderately resistant ( 5 to $8 \mathrm{~mm}), \mathrm{n}=11$

Ying Jy Horong Shin, TTES No.17, Ch5351, Ch5354, Ch5359, Ch5401, Ch5410, Makura-Ck2, Makura-Cm18, Makura-Cn14, Makura-Cp16

Susceptible (over $8 \mathrm{~mm}$ ), $\mathrm{n}=2$

Makura-Cd109, Miya-Ck68

Cultivars from China: Tiee Guan In, Chin Shin Oolong, Hwang Gan, Wuu Yi, San Cha Jy Lan, Chin Shin Dah Pan,

Dah Yeh Oolong, Jwu Yeh, Ying Jy Horong Shin, TTES No.17. 
Table 4. Classification of anthracnose resistance among tea genetic resources of Japan

\begin{tabular}{|c|}
\hline Highly resistant (lesion $<3 \mathrm{~mm}$ ), $\mathrm{n}=15$ \\
\hline $\begin{array}{l}\text { Yamatomidori, Tsukasamidori, KM8, KM62, Benihomare, Indo, Benitachiwase, Benikaori, Benihikari, Benifuuki, MAKURA lgou, } \\
\text { Makurazaki Igou, Makurazaki 2gou, Makurazaki 4gou, Makurazaki } 5 \text { gou }\end{array}$ \\
\hline Resistant (lesion 3 to $5 \mathrm{~mm}$ ), $\mathrm{n}=25$ \\
\hline $\begin{array}{l}\text { Fujisawabansei, Kousyun, Kuramochiwase, Kuramochibannsei, Marishi, S6, ME81, MO6, MO133, Yamanami, Unkai, } \\
\text { Minamikaori, Hatsumomiji, Akane, Benifuji, Satsumabeni, Minamisayaka, Tadanishiki, Shizu4006, Fukuin2, Fukuin7, A5, } \\
\text { Makurazaki 15gou, Makurazaki 16gou, Miya-F1-9-4-48 }\end{array}$ \\
\hline Moderately resistant ( 5 to $8 \mathrm{~mm}), \mathrm{n}=50$ \\
\hline $\begin{array}{l}\text { Asatsuyu, Makinoharawase, Takachiho, Natsumidori, Himemidori, Kanayamidori, Meiryoku, Fukumidori, Saemidori, Sainomidori, } \\
\text { Harumoegi, Miyamakaori, Yutakamidori, Samidori, Satouwase, NN27, Ujimidori, Inaguchi, Kuritawase, Shizuzai16, Kanaya } \\
\text { 25gou, Kanaya 27gou, Kanaya 28gou, Miyazaki 19gou, Miyazaki 23gou, Miyazaki 25gou, Miyazaki 26gou, Miyazaki 27gou, } \\
\text { Miyazaki 28gou, Saitama 35gou, Saitama 29gou, Saitama 42gou, Shigeru 2gou, ME3, ME29, ME11, ME71, MO29, MO218, } \\
\text { Musashikaori, Ryofu, Soufu, Houryoku, Fujimidori, Shizu-Inzatsu131, Sawamizuka, Fujikaori, Makurazaki 13gou, Makurazaki } \\
\text { 23gou, Kanaya 22gou }\end{array}$ \\
\hline Susceptible (over $8 \mathrm{~mm}$ ), $\mathrm{n}=52$ \\
\hline $\begin{array}{l}\text { Miyoshi, Tamamidori, Sayamamidori, Yabukita, Koyanishi, Rokurou, Yaeho, Asagiri, Kyomidori, Hatsumidori, Okumusashi, } \\
\text { Sayamakaori, Okumidori, Toyoka, Okuyutaka, Shyunmei, Minekaori, Fusyun, Hokumei, Sakimidori, Harumidori, Asahi, Ooiwase, } \\
\text { Surugawase, Terakawawase, Yamakai, Yamanoibuki, Komakage, Kurasawa, Z1, Mie260, NN12, Shika999, Ureshino 1gou, } \\
\text { Mieryokumei 1gou, Nagasaki 2gou, ME2, ME52, Gruszynski8, Makurazaki 19gou, Makurazaki 21gou, Kanaya 24gou, Kanaya } \\
\text { 26gou, Miyazaki 21gou, Miyazaki 22gou, Saitama 36gou, Saitama 41gou, Asanoka, Okuhikari, Izumi, Makurazaki 12gou, } \\
\text { Miyazaki 24gou }\end{array}$ \\
\hline
\end{tabular}

Normal: Crossbreeds of Japanese native tea plants. Bold: Crossbreeds of introduced Chinese varieties with Japanese tea plants. Italic: Crossbreeds of Assam varieties with Japanese tea plants.

dori' showed high resistance. Twenty-five of the Japanese tea plants showed anthracnose resistance, although 13 of them were crosses between Japanese tea plants and the Assam variety or introduced Chinese varieties. Fifty Japanese genetic resources showed moderate anthracnose resistance, and most of these plants were bred from native Japanese tea plants. At the same time, 52 Japanese genetic resources were susceptible to anthracnose, and most of these plants were also bred from native Japanese tea plants. All of the high-quality and high-yield Japanese tea cultivars such as 'Saemidori' and 'Sakimidori' were estimated to have moderate resistance or to be susceptible to anthracnose.

\section{Discussion}

Tea anthracnose is one of the most serious tea pests in Japan. Since most green tea cultivars in Japan are susceptible to anthracnose, the breeding of anthracnose resistant tea is required. In the present study, we assayed 500 tea genetic resources by wound-inoculation assay to search for materials of tea anthracnose resistance.

Most of the Assam plants showed high resistance and resistance, 14 plants showed moderate resistance, and no plants were susceptible. All pure Assam varieties showed high resistance or resistance. These plants were introduced from low latitude areas where the climate is warm with high humidity. It is assumed that tea plants in low latitudes had been culled by anthracnose infection for a long time, with only resistant tea plants surviving. On the other hand, the Assam varieties are not hardy in cold climates $^{7}$. Moreover, pure Assam variety plants contain high amounts of catechins and caffeine ${ }^{7}$, making them suitable for black tea but not Japanese green tea. Therefore, to produce high-quality cultivars with anthracnose resistance, crosses should be made between the Assam variety and Japanese cultivars.

Most of the introduced Chinese varieties showed high resistance and resistance, 11 plants showed moderate resistance and 'Miya-Ck68' and 'Makura-Cd109' showed susceptibility. Tea genetic resources of Miya-Ck were introduced from Anhui Province, China. While tea genetic resources of Makura-Cd were introduced from India, tea plants of $C$. sinensis var. sinensis in this area might have been introduced from China before the 20th century. The other tea genetic resources introduced from southwest areas of China such as Anhui Province, Hubei Province, Jiangxi Province, and Zhejiang Province 
showed wide variations of resistance, but none was susceptible. Tested Korean tea plants as well showed only resistance in this experiment.

On the other hand, Ikeda and Park ${ }^{4}$ reported that Korean tea genetic resources showed wide variation of resistance to anthracnose in field observations. However, since the number and introduced areas of Korean tea genetic resources in Makurazaki are limited, the variation of resistance of Korean tea was not considered in this study.

These results suggest that the plants of introduced Chinese varieties are good for breeding materials of anthracnose resistant tea. In addition, the green tea quality of introduced Chinese varieties is better than that of Assam varieties and they are hardy in cold climates. Therefore, introduced Chinese plants can be used to breed anthracnose-resistant tea plants with a taste suitable for the Japanese market.

Japanese tea genetic resources consist of Japanese tea cultivars and native tea plants that have been collected from across Japan. Japanese tea cultivars contain green tea cultivars, semi-fermented tea cultivars and black tea cultivars. A number of Japanese genetic resources that are crossbreeds of Japanese native plants and Assam or introduced Chinese varieties showed high resistance and resistance. However, the green tea quality of these resistant Japanese tea genetic resources was inferior to that of 'Yabukita'.

On the other hand, the tea genetic resources bred from Japanese native tea plants showed only moderate resistance or even susceptibility although the quality of green tea was good. Matsumoto et al. ${ }^{5}$ reported that RFLP analysis indicated the genetic diversity of Japanese tea is less than that of tea genetic resources from China. Green tea plants were first introduced from China to Japan in the 9th century. The areas of China exporting tea to Japan were restricted, so, tea plants with narrow genetic diversity were introduced and developed in $\mathrm{Japan}^{5}$. Some of the introduced Chinese varieties showed susceptibility and moderate resistance in this study. It is assumed that high quality for Japanese green tea but anthracnose susceptible tea plants were introduced and developed in Japan. Japanese tea cultivars have been bred from a small number of tea plants and 'Yabukita', therefore, genetic diversity of Japanese tea is restricted and a number of tea plants were found to be anthracnose susceptible.

The anthracnose resistance gene of tea is unknown. Toyao et al. ${ }^{10}$ revealed that heredity of anthracnose resistance didn't follow Mendelian inheritance, the heritability of anthracnose resistance is high and some quantitative resistance genes might determine the degree of resistance. Ikeda and Anma's ${ }^{3}$ diallel analysis of tea anthracnose resistance revealed that it was controlled by an additive effect of some quantitative genes of anthracnose resistance. These results suggest that breeding for anthracnose resistance can be done by using quantitative resistance genes. Our wound-inoculation assay would be useful for selecting anthracnose resistant tea plants.

The monoculture of 'Yabukita' has caused severe pest problems in Japan. Tea gray blight caused by Pestalotiopsis longiseta and white peach scale (Pseudaulacaspis pentagona) are destructive pests of 'Yabukita' and other Japanese tea cultivars as well as anthracnose. For resistance breeding, it is very important to develop methods for selecting resistant materials. Methods for selecting resistant breeding materials of tea gray blight and white peach scale have already been developed $^{9,12}$. These methods and our method will be useful for developing a pest-resistant tea breeding system. By use of three pest-resistance assays, we selected 'KM8', 'KM62' and 'Minamisayaka' for the parents of anthracnose, gray blight and white peach scale resistant tea breeding. 'KM8' and 'KM62' are crossbreeds of 'Sayamakaori' and 'Kana-Ck17'. 'Minamisayaka' is a crossbreed of an Assam variety and a Japanese cultivar. These plants were bred from Japanese native plants and foreign-introduced tea genetic resources. Thus, the crossbreeding of Japanese tea cultivars with foreign introduced varieties would be useful for breeding pestresistant tea plants of suitable quality. We are trying to breed high quality, high-yield and multiple-pest resistant tea by using the above three tea plants.

\section{References}

1. Ezuka, A. \& Ando, Y. (1994) Anthracnose. In Cha no byougai (Tea dis. in Jpn.), eds. Ezuka, A.\& Ando, Y., Japan Plant Protection Association, Tokyo, Japan, 182204 [In Japanese].

2. Hamaya, E. (1982) Trichome infection of the tea anthracnose fungus Gloeosporium theae-sinensis. JARQ, 16, 114-118.

3. Ikeda, N. \& Amma, S. (2004) Diallel analysis of resistance to anthracnose in tea (Camellia sinensis). Ikushugaku Kenkyu (Breed. Res.), 6, 135-141 [In Japanese with English summary].

4. Ikeda, N. \& Park, Y. -G. (2005) Resistance of Korean tea landrace populations, to tea anthracnose, tea gray blight and tea bacterial shoot blight. Ikushugaku Kenkyu (Breed. Res.), 7, 35-39 [In Japanese].

5. Matsumoto, S., Kiriiwa, Y. \& Takeda, Y. (2002) Differentiation of Japanese green tea cultivars as revealed by RFLP analysis of phenylalanine ammonia-lyase DNA. Theor. Appl. Genet., 104, 998-1002.

6. Oniki, M., Hamaya, E. \& Ando, Y. (1985) The actual note of resistant strains of the tea anthracnose fungus 
Gloeosporium theae-sinensis against benzimidazole fungicides in Japan. Chagyou Kenkyu Houkoku (Tea Res. J.), 61, 7-11 [In Japanese with English summary].

7. Takeda, Y. (2002) Studies on variations in genetic resources of tea in Japan and application to tea breeding. Bull. Natl. Inst. Veg. \& Tea Sci., 1, 97-180 [In Japanese with English summary].

8. Takeda, Y. \& Yoshida, K. (2002) Evaluation of tea genetic resources in the resistance to gray blight and anthracnose. Ikushugaku Kenkyu (Breed. Res.), 4, (Suppl. 2), 306 [In Japanese].

9. Tanaka, J. (2005) Practical marker-assisted selection for mulberry scale resistance of tea and its future. Norinsuisangijutsu Kenkyu Journal (Res. J. of Food \& Agric.), 28(9), 39-44 [In Japanese].

10. Toyao, T., Takeda, Y. \& Matsushita, S. (1976) Varietal difference and heritability estimates for resistance to anthracnose disease in tea plant. Chagyou Gijutsu
Kenkyu (Stud. Tea), 50, 1-8 [In Japanese with English summary].

11. Yamaguchi, S. \& Ando, Y. (1993) Testing of tea anthracnose disease resistance among genetic resources for the disease resistance breeding of tea. Ikushugaku Zasshi (Jpn. J. of Breed.), 43, (Suppl. 1), 173 [In Japanese].

12. Yanase, Y. \& Takeda, Y. (1987) Method for testing the resistance to tea gray blight caused by Pestalotia longiseta Spegazzini in tea breeding. Bull. Natl. Res. Inst. Veg. Orn. Plants \& Tea, B 1, 1-9 [In Japanese with English summary].

13. Yoshida, K. \& Takeda, Y. (2004) Assay method for screening of tea plants with resistance to anthracnose caused by Colletotrichum theae-sinensis by use of novel wound-inoculation. Bull. Natl. Inst. Veg. \& Tea Sci., 3, 137-146 [In Japanese with English summary]. 